\title{
Current application of metabolomics in the elucidation of processing mechanisms used in Chinese materia medica: A review
}

\author{
Ting Huang ${ }^{1}$, Yunbin Jiang ${ }^{2}$, Yanfei Zhang ${ }^{3}$, Yutian Lei ${ }^{1}$, Guihua Jiang ${ }^{1 *}$ \\ ${ }^{1}$ College of Pharmacy, Chengdu University of Traditional Chinese Medicine, Chengdu 611137, ${ }^{2}$ College of Pharmaceutical \\ Sciences and Chinese Medicine, Southwest University, Chongqing 400715, ${ }^{3}$ Institute of Tibetan medicine, Tibetan Traditional \\ Medical College, Lhasa 850000, China
}

*For correspondence: Email: 11469413@qq.com; Tel: +86-18980923782

Sent for review: 11 November 2019

Revised accepted: 18 May 2020

\begin{abstract}
Processing, a key characteristic of traditional Chinese medicine (TCM), enhances the efficacy and safety of Chinese materia medica (CMM) in clinics. It plays an important role in TCM. Studies on processing mechanisms involved in CMM promote the development of TCM. However, most studies on the mechanisms used for processing CMM do not reflect the holistic theory of TCM because they are based only on analysis of some specific chemical components and biochemical indices which do not support the TCM characteristics of network target and multicomponent therapeutics. Fortunately, the perspective of systems biology is consistent with the holistic theory of TCM. Metabolomics, a key tool in systems biology, has been widely used to investigate the processing mechanism of CMM for many years. In this work, current applications of metabolomics in elucidating mechanisms used for processing of CMM were systematically reviewed and discussed in terms of changes in chemical components, toxicity and efficacy of CMM before and after processing. This work provides researchers a clear and concise reference on the current application of metabolomics in investigation of mechanisms used in processing of CMM. Moreover, this work provides a guide on how to investigate the mechanisms used in processing of CMM, based on metabolomics.
\end{abstract}

Keywords: Chinese materia medica, Processing mechanism, Metabolomics, Holistic theory

\begin{abstract}
This is an Open Access article that uses a fund-ing model which does not charge readers or their institutions for access and distributed under the terms of the Creative Commons Attribution License (http://creativecommons.org/licenses/by/4.0) and the Budapest Open Access Initiative (http://www.budapestopenaccessinitiative.org/read), which permit unrestricted use, distribution, and reproduction in any medium, provided the original work is properly credited.
\end{abstract}

Tropical Journal of Pharmaceutical Research is indexed by Science Citation Index (SciSearch), Scopus, International Pharmaceutical Abstract, Chemical Abstracts, Embase, Index Copernicus, EBSCO, African Index Medicus, JournalSeek, Journal Citation Reports/Science Edition, Directory of Open Access Journals (DOAJ), African Journal Online, Bioline International, Open-J-Gate and Pharmacy Abstracts

\section{INTRODUCTION}

Unlike Western herbs, most Chinese materia medica (CMM) may be steamed, fried, charred, boiled, or treated with excipients such as vinegar or yellow rice wine before they are used in manufacturing CMM products and decoctions [1]. These specific treatments are referred to as processing (Paozhi in Chinese), and they are ancient Chinese pharmaceutical techniques for enhancing the clinical application of CMM [2].

The main processing methods for CMM, as outlined in the Chinese pharmacopoeia (2015 edition), are summarized in Table 1. 
Table 1: Main processing methods of $\mathrm{CMM}$ and corresponding representative $\mathrm{CMM}$ in the Chinese pharmacopoeia (2015 edition)

\begin{tabular}{|c|c|c|}
\hline Method & Excipient & Representative CMM \\
\hline Stir-frying & - & Arecae Semen \\
\hline \multirow[t]{4}{*}{ Stir-frying with solid excipient } & Sand & Strychni Semen \\
\hline & Bran & Aurantii Fructus \\
\hline & Rice & Mylabris \\
\hline & Fine powders of terra & Dioscoreae Rhizoma \\
\hline \multirow[t]{6}{*}{ Stir-frying with liquid excipient } & Vinegar & Kansui Radix \\
\hline & Salt-water & Eucommiae Cortex \\
\hline & Yellow rice wine & Angelicae Sinensis Radix \\
\hline & Ginger juice & Tsaoko Fructus \\
\hline & Refined honey & Lilii Bulbus \\
\hline & Refined suet & Epimedii Folium \\
\hline \multirow[t]{5}{*}{ Steaming } & Steaming only & Polygoni Multiflori Radix \\
\hline & Vinegar & Schisandrae Chinensis Fructus \\
\hline & Salt-water & Morindae Officinalis Radix \\
\hline & Yellow rice wine & Rhei Radix et Rhizoma \\
\hline & Medicinal juice & Polygoni Multiflori Radix \\
\hline \multirow[t]{4}{*}{ Boiling } & - & Aconiti Radix \\
\hline & $\begin{array}{l}\text { Edible mother liquor of } \\
\text { mineral salt }\end{array}$ & Aconiti Lateralis Radix Praeparata \\
\hline & $\begin{array}{l}\text { Zingiberis Rhizoma } \\
\text { Recens and alumen }\end{array}$ & Pinelliae Rhizoma \\
\hline & $\begin{array}{l}\text { Glycyrrhizae Radix et } \\
\text { Rhizoma and lime }\end{array}$ & Pinelliae Rhizoma \\
\hline Calcining & - & Gypsum Fibrosum \\
\hline Water trituration & - & Cinnabaris \\
\hline
\end{tabular}

The major objectives of CMM processing are attenuation of toxicity and enhancement of efficacy. Indeed, changes in chemical components of CMM during processing are responsible for the changes in toxicity and efficacy of CMM after processing [3]. Nowadays, several studies have determined the effect of processing on chemical components, toxicity and efficacy of CMM. However, the perspectives of most of these studies do not reflect the holistic theory of traditional Chinese medicine (TCM) because they involve investigations of the effect of processing on toxicity and efficacy of CMM based only on measurements of some specific chemical components and biochemical indices $[4,5]$. This is at variance with the characteristics of TCM i.e. network target and multicomponent therapeutics [6,7]. Therefore, these studies are not adequate for elucidating the mechanisms involved in the processing of CMM based on the holistic theory of TCM. For example, it was reported that the toxicity and efficacy of areca nut, a frequently-used CMM, were attenuated and increased, respectively, after processing, due to changes in some specific components and biochemical indices such as arecoline, cell/animal toxicity, and gastrointestinal tract functions $[8,9]$. Obviously, these indices do not reflect network target and multicomponent therapeutics, which are the characteristics of TCM. Thus, these studies cannot elucidate the mechanism used in processing areca nut based on the holistic theory of TCM.

The perspective of systems biology, which is consistent with the holistic theory of TCM, has been widely used to study TCM-related issues such as chemical components, toxicity and efficacy of CMM [10-12]. At present, omics techniques have become the key tools of systems biology, with metabolomics constituting an important part [13]. Metabolomics research procedures comprise three steps: sample collection and preparation, metabolome data collection, and data analysis $[14,15]$. Metabolomics has been widely used for investigation of mechanisms used in the processing of CMM for many years [16]. In the present study, the applications of metabolomics in elucidating mechanisms used in processing of CMM were systematically reviewed and discussed in terms of the changes in chemical 
components, toxicity and efficacy of CMM before and after processing. This was with a view to enhancing the investigations on mechanisms used for processing of CMM based on metabolomics.

\section{DECIPHERING THE EFFECT OF PROCESSING ON CHEMICAL COMPONENTS OF CMM, BASED ON METABOLOMICS}

The effect of processing on chemical components of CMM can been comprehensively determined using metabolomics. Metabolomics can be used for direct demonstration of the effect of processing on chemical component profiles of CMM. The differences in chemical component profiles of CMM before and after processing consist of changes in contents of components, production of new components, and elimination of original components. The biomarkers of processing-related chemical components identified using multivariate statistical analysis of metabolomics data can be used to further elucidate precisely the effect of processing on chemical components of CMM.

It has been reported that after steaming or boiling with vinegar or yellow rice wine, the results of metabolomics analysis indicated marked changes in chemical component profile of Ligustri Lucidi Fructus, with significant increase in the content of processing-related chemical component biomarker (ligustaloside B) [17]. Following stir-frying with refined honey or licorice juice, the chemical component profile of Polygalae Radix exhibited marked changes, based on metabolomics analysis [18]. Metabolomics analysis revealed that the levels of 127 components of Rhubarb were changed after it was steamed with yellow rice wine [19]. There are many similar studies in the literature, such as the effect of processing on the chemical components of Schisandrae Chinensis Fructus, Euphorbiae Pekinensis Radix, and Asari Radix et Rhizoma [20-22].

\section{METABOLOMICS-BASED ELUCIDATION OF THE EFFECT OF PROCESSING ON TOXICITY OF CMM}

Changes in chemical components of CMM are the underlying causes of changes in toxicity of CMM after processing. Generally, the effect of processing on toxicity of CMM is investigated based on metabolomics by comparing the differences in endogenous metabolites of experimental animals treated with crude CMM and processed CMM. To better elucidate the effect of processing on toxicity of CMM, changes in chemical components of CMM before and after processing should be investigated simultaneously. However, extant literature suggests that the current application of metabolomics in elucidating the attenuating effect of processing on toxicity of CMM has focused mainly on analysis of endogenous metabolites of organism. The processing-related endogenous metabolite biomarkers and chemical component biomarkers identified using multivariate statistical analysis of metabolomics data can be used to further elucidate precisely the attenuating effect of processing on toxicity of CMM.

After being processed with sand or oil, the results of metabolomics analysis showed obvious differences in the chemical component profile of Strychni Semen: its main toxic and bioactive components strychnine and brucine were converted to less-toxic and equally-active components strychnine $\mathrm{N}$-oxide and brucine $\mathrm{N}$ oxide, respectively [23]. The liver and kidney toxicities of Myristicae Semen were decreased after processing with wheat bran, and the results of metabolomics analysis indicated significant reversal (to normal) of the Myristicae Semeninduced abnormality in rat endogenous metabolite profile. Moreover, 17 endogenous metabolites were identified as biomarkers related to processing-induced attenuation of Myristicae Semen toxicity [24].

A study has demonstrated that, after nine sessions of steaming and sunning, the liver toxicity of Polygoni Multiflori Radix was attenuated. Moreover, metabolomics analysis revealed that Polygoni Multiflori Radix-induced changes in rat endogenous metabolite profile were significantly reversed to normal [25]. In addition, 10 metabolites were identified as biomarkers related to processing-induced attenuation of Polygoni Multiflori Radix toxicity [25]. It has been reported that processing reduced the toxicity of Pinelliae Rhizoma, and results of metabolomics analysis suggested that processing significantly reversed Pinelliae Rhizoma-induced abnormality in rat endogenous metabolite profile [26]. Moreover, 10 metabolites were identified as biomarkers related to processing-induced reduction in the toxicity of Pinelliae Rhizoma [26].

\section{METABOLOMICS-BASED INVESTIGATON OF THE EFFECT OF PROCESSING ON EFFICACY OF CMM}

Similar to their influence on toxicity of CMM, changes in chemical components of CMM also result in changes in efficacy of CMM after processing. The major objectives of CMM 
processing are to increase original efficacy, and to produce new efficacy. Simultaneous analyses of changes in chemical components of CMM before and after processing, and comparison of the differences in endogenous metabolites of experimental animals treated with crude CMM and processed CMM would enhance elucidation of the effect of processing on efficacy of CMM. However, studies on the effect of processing on efficacy of CMM have focused mainly on analyses of endogenous metabolites of organisms, with very few chemical component analyses. Similarly, processing-related endogenous metabolite biomarkers and chemical component biomarkers can be used to determine precisely the effect of processing on efficacy of CMM.

A study has shown that after stir-frying with vinegar, the liver-soothing and analgesic efficacies of Bupleuri radix were enhanced, and Bupleuri Radix-induced rat endogenous metabolite profile was significantly changed, based on metabolomics analysis, suggesting obvious differences in the efficacy of Bupleuri radix before and after processing [27]. The major processed products of Angelicae sinensis Radix (ASR), which have been widely used in TCM prescriptions, are charred ASR, oil-parched ASR, wine-parched ASR, and soil-parched ASR. It has been reported that charred ASR and wineparched ASR produced better anti-inflammatory effects than ASR parched with oil or soil, and metabolomics analysis suggested that charred ASR and wine-parched ASR were better at reversing abnormality of endogenous metabolite profiles of rats with acute inflammation, than oilor soil-parched ASR [28].

There are many similar metabolomics-based studies on the effect of different processing methods on the efficacy of ASR [29,30]. Raw Vladimiriae Radix promotes the circulation of qi, but the efficacy was converted to anti-diarrhea effect after simmer processing. Results of metabolomics analysis have suggested that the chemical component profile of Vladimiriae Radix was different after processing, and 7 chemical components were identified as biomarkers related to processing-induced changes in Vladimiriae Radix efficacy [31]. Based on metabolomics study, the differential influences of Psoraleae fructus and salt-processed Psoraleae fructus on 22 common biomarkers and associated metabolic pathways showed that saltprocessing enhanced the effect of Psoraleae fructus and reduced its cardiovascular and renal toxicities in rats [32].
It has been reported that wine-processed Scutellariae radix (WSR) exhibited a more remarkable mitigating effect on acute lung injury than crude Scutellariae Radix (CSR). Pathway analysis of metabolomics data indicated that CSR acted on acute lung injury by regulating the metabolic pathway of abnormal sphingolipids, but WSR-mediated treatment was related mainly to reversal (to normal) of the abnormality in retinol and tryptophan metabolic pathways. These findings suggested that wine processing changed the mechanism of action of Scutellariae radix against acute lung injury [33]. There are many similar studies on the effect of processing on efficacy of CMM, based on metabolomics. These include effect of processing on the efficacies of Gardeniae Fructus, Zingiberis Rhizoma, Rehmanniae Radix, and Codonopsis Radix [3437].

\section{METABOLOMICS-BASED ELUCIDATION OF CMM PROCESSING MECHANISM FROM CHEMICAL COMPONENTS, TOXICITY AND EFFICACY}

At present, the investigations on processing of CMM focus mainly on elucidating the effects of processing methods on chemical components, toxicity and efficacy of CMM [38]. Processinginduced changes in chemical components of CMM result in changes in toxicity and efficacy of CMM. The effect of processing on the chemical component profiles of CMM, and CMM-induced endogenous metabolite profiles of experimental animals can be comprehensively determined using metabolomics. Chemical component and endogenous metabolite profiles can be used to show the holistic differences in chemical components, toxicity and efficacy of CMM before and after processing. The differences can be further elucidated through chemical component biomarkers and endogenous metabolite biomarkers identified using multivariate statistical analysis of metabolomics data.

Endogenous metabolite biomarkers can be used to elucidate the pathways associated with the differences in toxicity and efficacy of CMM before and after processing, with the aid of Kyoto Encyclopedia of Genes and Genomes [39]. In brief, the mechanisms involved in attenuation of toxicity and enhancement of efficacy in CMM processing can be elucidated through analysis of the differences in the chemical components of CMM and CMM-induced endogenous metabolites of experimental animals before and after processing, with the aid of metabolomics. However, the current application of metabolomics in CMM processing shows that the relationships between chemical components and 
toxicity/efficacy of CMM are not well analyzed. As a result, studies on the mechanisms used in processing of CMM are not in-depth enough: they require further enhancement.

\section{FINAL REMARKS}

This review has systematically summarized the current application of metabolomics in studies on the effect of processing on chemical components, toxicity and efficacy of CMM. The approaches to elucidating processing mechanism of CMM based on metabolomics are presented. This work provides a clear and concise reference for researchers on the mechanisms used for processing CMM and their effects on chemical components, toxicity and efficacy, based on metabolomics.

\section{DECLARATIONS}

\section{Acknowledgement}

This work was financially supported by the Academic Backbone Project of "Xinglin Scholars" (no. XSGG-2019023), the Young Scholars Project of "Xinglin Scholars" (no. QNXZ2019024), and the Poverty Alleviation Project of Science and Technology Department of Sichuan Province (no. 2018NFP0112).

\section{Conflict of interest}

No conflict of interest is associated with this work.

\section{Contribution of authors}

We declare that this work was done by the authors named in this article and all liabilities pertaining to claims relating to the content of this article will be borne by the authors. All authors have read and approved the publication of manuscript. Guihua Jiang and Yunbin Jiang conceived and designed the study, while Ting Huang, Yanfei Zhang and Yutian Lei collected and analyzed the literatures. Ting Huang and Yunbin Jiang wrote the manuscript. Ting Huang and Yunbin Jiang contributed equally to this work and should be considered as co-first authors.

\section{Open Access}

This is an Open Access article that uses a funding model which does not charge readers or their institutions for access and distributed under the terms of the Creative Commons Attribution License (http://creativecommons.org/licenses/by/
4.0) and the Budapest Open Access Initiative (http://www.budapestopenaccessinitiative.org/rea d), which permit unrestricted use, distribution, and reproduction in any medium, provided the original work is properly credited.

\section{REFERENCES}

1. Zhao Z, Liang Z, Chan K, Lu G, Lee EL, Chen H, Li L. A unique issue in the standardization of Chinese Materia Medica: processing. Planta Med 2010; 76(17): 19751986.

2. Wu X, Wang S, Lu J, Jing Y, Li M, Cao J, Bian B, Hu C. Seeing the unseen of Chinese herbal medicine processing (Paozhi): advances in new perspectives. Chin Med 2018; 13: 4.

3. Ou SP, Wang S, Yang QY, Chen ZH, Wu HM, Yang $M$. Advances in studies on dried resin of Garcinia hanburyi processing in toxicity reducing and efficacy enhancing effects. Chin Tradit Herb Drugs 2011; 42(11): 25602563. (in Chinese)

4. Tu C, Jiang BQ, Zhao YL, Li CY, Li N, Li XF, Jia GLC, Pang JY, Ma ZJ, Wang JB, et al. Comparison of processed and crude Polygoni Multiflori Radix induced rat liver injury and screening for sensitive indicators. Chin J Chin Materia Medica 2015; 40(4): 654-660. (in Chinese)

5. Huang $Y F$, Sun $M, X u Y Z$, Wang JX, Zhao $X Y$, Chen $K L$, Huang BS, Liu $Y$. Influence of different processed methods on 10 kinds of ginsensides in Panacis Quinquefolii Radix. Chin J Chin Materia Medica 2014; 39(20): 3950-3954. (in Chinese)

6. Jiang $Y$, Zhong $M$, Long $F$, Yang $R$. Deciphering the active ingredients and molecular mechanisms of Tripterygium hypoglaucum (Levl.) Hutch against rheumatoid arthritis based on network pharmacology. Evid Based Complement Alternat Med 2020; 2020: 2361865.

7. Jiang $Y$, Zhong $M$, Long $F$, Yang $R$, Zhang $Y$, Liu $T$. Network pharmacology-based prediction of active ingredients and mechanisms of Lamiophlomis rotata (Benth.) Kudo against rheumatoid arthritis. Front Pharmacol 2019; 10: 1435.

8. Peng W, Liu YJ, Hu MB, Yan D, Gao YX, Wu CJ. Using the "target constituent removal combined with bioactivity assay" strategy to investigate the optimum arecoline content in charred areca nut. Sci Rep 2017; 7: 40278.

9. Li K, Wang W, Yan X, Wu C, Ye L. Impact of Maillard reaction on the arecoline transformation during Arecae semen parching process. West Chin J Pharmaceut Sci 2017; 32(2): 150-153. (in Chinese)

10. Li P, Yang LP, Gong YW. Application of systems biology technology in research of traditional Chinese medicine. J Tradit Chin Med 2009; 29(2): 153-157.

11. Zhang JY, Wang QG, Wang Z. Systems biology and traditional Chinese medicine (TCM) modernization. Chin J Chin Materia Medica 2005; 1725-1727, 1766. (in Chinese)

Trop J Pharm Res, June 2020; 19(6): 1325 
12. Liu P, Liu S, Tian D, Wang P. The applications and obstacles of metabonomics in traditional Chinese medicine. Evid Based Complement Alternat Med 2012; 2012: 946824

13. Buriani A, Garcia-Bermejo ML, Bosisio E, Xu Q, Li H, Dong $X$, Simmonds MS, Carrara M, Tejedor N, LucioCazana J, et al. Omic techniques in systems biology approaches to traditional Chinese medicine research: present and future. J Ethnopharmacol 2012; 140(3): 535-544.

14. Dunn WB, Ellis DI. Metabolomics: current analytical platforms and methodologies. Trac-Trend Anal Chem 2005; 24(4): 285-294.

15. Hu C, Xu G. Metabolomics and traditional Chinese medicine. Trac-Trend Anal Chem 2014; 61: 207-214.

16. Chen Z, Hu C, Zheng W, Jin Y, Cao D, Wu S. Overview on application of metabonomics in study on processing mechanism of traditional Chinese materia medica. Chin Arch Tradit Chin Med 2018; 36(2): 275-279. (in Chinese)

17. Guo N, Fan B, Peng J, Yan H, Ma F, Yu YH. Ultraperformance LC/TOF MS analysis of fruits of Ligustrum lucidum for metabolomic research. Chin J Exper Tradit Med Formu 2010; 16(10): 131-133. (in Chinese)

18. Wang XJ, Li ZY, Xue SY, Zhang FS, Xing J, Qin XM. Quality control over different processed products of Polygalae Radix based on plant metabolomics. Chin Tradit Herb Drugs 2012; 43(9): 1727-1737. (in Chinese)

19. Zhao N, Zhang XZ, Hu CJ, Jia TZ, Xiao HB. Metabolomics analysis revealing multiple compounds changed in rhubarb after processing. Chin J Chin Materia Medica 2014; 39(9): 1607-1613. (in Chinese)

20. Li W, Song YG, Liu KY, Yang LJ, Liu YL, Su D, Feng YL. Rapid identification of the different constituents in Fructus Schisandrae Chinensis before and after processing by UHPLC-QTOF/MSE combining with metabonomics. Acta Pharm Sinica 2016; 51(9): 14451450. (in Chinese)

21. Zeng $Y$, Hou PY, Chen $X H$. Investigation of variations of components in Euphorbia pekinensis Root and its processed products based on plant metabolomics. J Chin Med Mater 2016; 39(3): 530-533. (in Chinese)

22. Li Y, Fan ML, Xing J, Qin XM, Li ZY. Metabolomic studies on special processing of Asari Radix et Rhizoma in Guilingji. Chin Tradit Herb Drugs 2015; 46(16): 23852393. (in Chinese)

23. Wu W, Qiao C, Liang Z, Xu H, Zhao Z, Cai Z. Alkaloid profiling in crude and processed Strychnos nux-vomica seeds by matrix-assisted laser desorption/ionizationtime of flight mass spectrometry. J Pharm Biomed Anal 2007; 45(3): 430-436.

24. Wang J, Chen Y, Yuan ZM, Jia TZ. Differences Studied on mechanism of long-term toxicity in rats of Myristicae Semen after and before. Chin J Exper Tradit Med Formu 2018; 24(4): 8-13. (in Chinese)

25. Li CY, He Q, Tang JF, Sha MC, Tu C, Zhang L, Liu ZX. Metabolomic study on immunological stress-mediated hepatotoxicity of Polygonum multiflorum and its processed products of nine times steaming and nine times sunning. Acta Pharm Sinica 2017; 52(7): 10691076. (in Chinese)

26. Su T, Tan Y, Tsui MS, Yi H, Fu XQ, Li T, Chan CL, Guo $H$, Li YX, Zhu PL, et al. Metabolomics reveals the mechanisms for the cardiotoxicity of Pinelliae Rhizoma and the toxicity-reducing effect of processing. Sci Rep 2016; 6: 34692.

27. Dai J, Zheng N, Sun S, Fang J, Wang Y, Li Q, Cao H, Zhao J, Zhang $Y$. Research on biological effects of vinegar stir-frying method on radix bupleuri chinensis by serum metabonomics. Chin Arch Tradit Chin Med 2014; 32(07): 1717-1720. (in Chinese)

28. Zhong $L J$, Zhang WQ, Hua YL, Ji P, Yao WL, Li J, Li PL, Wei $Y M$. Metabolomic evaluation for anti-inflammatory effect of volatile oils from different preparations of Angelicae Sinensis Radix. Chin J Chin Materia Medica 2016; 41(11): 2061-2069. (in Chinese)

29. Yuan Z, Zhong L, Hua Y, Ji P, Yao W, Ma $Q$, Zhang $X$, Wen $Y$, Yang $L$, Wei $Y$. Metabolomics study on promoting blood circulation and ameliorating blood stasis: Investigating the mechanism of Angelica sinensis and its processed products. Biomed Chromatogr 2019; 33(4): e4457.

30. Hua $Y$, Xue W, Zhang $M$, Wei $Y$, Ji P. Metabonomics study on the hepatoprotective effect of polysaccharides from different preparations of Angelica sinensis. J Ethnopharmacol 2014; 151(3): 1090-1099.

31. Gao F, Fu CM, Hu HL, Wang ZG, Fu S, Mao Q, Sheng $F Y$, Peng $W$. Difference of metabolic profiling of Vladimiriae Radix before and after simmer processing by HPLC-UV technology. Chin Tradit Herb Drugs 2013; 44(5): 547-551. (in Chinese)

32. Li K, Zhou N, Zheng XK, Feng WS, Li F, Zhang ZL, Lu $Y Q$. Quantitative analysis, pharmacokinetics and metabolomics study for the comprehensive characterization of the salt-processing mechanism of Psoraleae Fructus. Sci Rep 2019; 9(1): 661.

33. Hu L, Wang $Y$, Sun $H$, Xiong $Y$, Zhong L, Wu Z, Yang $M$. An untargeted metabolomics approach to investigate the wine-processed mechanism of Scutellariae radix in acute lung injury. J Ethnopharmacol 2020; 253: 112665.

34. Zhang $X$, Wang $Y$, Li S, Dai Y, Li X, Wang Q, Wang G, Ma $Y, G u X$, Zhang $C$. The potential antipyretic mechanism of Gardeniae Fructus and its heatprocessed products with plasma metabolomics using rats with yeast-induced fever. Front Pharmacol 2019; 10: 491.

35. Mo MY, Zhu QH, Xue XY, Deng XM, Zhou SJ, Meng J, Wang SM. Urine metabonomics analysis of dried and charred Zingiberis Rhizoma Recens on rats with deficiency cold hemorrhagic disease. Chin J Exper Tradit Med Formu 2015; 21(16): 1-4. (in Chinese)

36. Xia F, Liu C, Wan JB. Characterization of the cold and hot natures of raw and processed Rehmanniae Radix by integrated metabolomics and network pharmacology. Phytomedicine 2019; https://doi.org/10.1016/j.phy med.2019.153071.

Trop J Pharm Res, June 2020; 19(6): 1326 
37. Hao YY, Nie CX, He P, Wu XW, Liu G, Hao XL. Effects of different processed products of Codonopsis pilosula on immunosuppressive rats based on $\mathrm{H}-1$ NMR metabolomics. Chin Pharmacol Bull 2019; 35(9): 12661273.

38. Sun E, Xu FJ, Zhang ZH, Jia XB. Discussion about research progress and ideas on processing mechanism of traditional Chinese medicine. Chin J Chin Materia Medica 2014; 39(3): 363-369. (in Chinese)

39. Wu Q, Zou $M$, Yang $M$, Zhou S, Yan X, Sun B, Wang $Y$, Chang S, Tang $Y$, Liang $F$, et al. Revealing potential biomarkers of functional dyspepsia by combining $1 \mathrm{H}$ NMR metabonomics techniques and an integrative multi-objective optimization method. Sci Rep 2016; 6: 18852. 